\title{
ATF5 Gene
}

National Cancer Institute

\section{Source}

National Cancer Institute. ATF5 Gene. NCI Thesaurus. Code C101599.

This gene is involved in the regulation of gene expression. 